\section{SUBJECT INDEX VOLUME 47, 2005}

${ }^{14} \mathrm{C}, 207-210,211-220,231-234,441-451$

${ }^{14} \mathrm{C}$ bomb peak, $115-134$

${ }^{14} \mathrm{C}$ dating, 11-20, 21-25, 89-98, 115-134, 221-230, 295-354

${ }^{14} \mathrm{C}$ intercomparison, 39-56, 359-366

${ }^{14} \mathrm{C}$ production, $31-38$

${ }^{14} \mathrm{CO}_{2}, 57-65$

$\Delta \mathrm{R}, 433-440$

Age-depth model, 115-134

Alaska (North), 359-366

Alkenones, 401-412, 413-424, 425-432

AMS, 207-210, 211-220, 221-230, 235-242, 377-382, 453-464

Archaeobotany, 377-382

Archaeology, 181-192, 265-294, 383-394, 395-400

Bayesian statistics, 147-158

Betelnut, 377-382

Blank corrections, 75-88

Bodega Bay, 265-294

Bomb ${ }^{14} \mathrm{C}$ models, 99-114

Bomb pulse, 27-30

Bone, 193-206, 367-375

Brazilian coast, 67-74

Calibration, 21-25, 115-134, 221-230

California, 265-294

Canarium, 377-382

Carbon blanks, 413-424

Carbon cycle, 31-38, 441-451

Carbon dioxide $\left(\mathrm{CO}_{2}\right), 257-263$

Catacombs, 395-400

Carbon turnover, 99-114

Cariaco Basin, 57-65

Channel Islands, 11-20

Charcoal, 193-206

Chronology, 147-158, 265-294, 395-400

$\mathrm{CO}_{2}$ vent, 257-263

Coastal migration, 383-394

Collagen, 193-206

Compound-specific, 413-424

Corals, 57-65

Cosmic rays, $31-38$

Dicarb, 359-366

Diet, 181-192, 367-375

Earthworks, 295-354

Gas analysis, 177-178

Gas tubes, 177-178

Graphitization, 453-464

High-precision, 89-98
High vacuum line, 453-464

Human bone, 181-192

Human impact, 159-176

Hunter-gatherers, 265-294

Ice wedges, 243-256

Industrial pollution, 135-146

Iron Age, 39-56

Isolation, 401-412

Italy, 27-30

Kurgans, 235-242

Lapita, 181-192

Lignite contamination, 1-10

Liquid scintillation, 89-98, 231-234

Marine reservoir, 67-74, 433-440

Marine sediments, 401-412, 413-424, 425-432

Micronesia, 295-354

Midden, 433-440

Neolithic chronology, 207-210

New Zealand, 367-375

Oceanic carbon, 75-88

Oregon, 383-394

Organic fractions, 75-88

OxCal, 147-158, 221-230

Palau, 1-10, 295-354

Paleosol, 159-176

Papua New Guinea, 377-382

Paraná, 67-74

Particulate organic matter (POM), 75-88

Peat dating, 135-146

Peat profiles, 115-134

Pollen, 243-256

Pottery dating, 1-10

Radon, 231-234

Raman and infrared spectrosophy, 193-206

Reconnaissance, 11-20

Reservoir effect, 67-74, 433-440

Rome, 395-400

Sample preparation, 211-220

San Miguel, 11-20

Santa Catarina, 67-74

Shag River Mouth site, 367-375

Spheroidal carbonaceous particles (SCPs), 135-146

Siberia, 243-256

Single-tube, 89-98

Small graphite targets, 453-464

SOC fractions, 99-114 
Soil dating, 159-176

Soil organic carbon, 99-114

Soil respiration, 441-451

Solvent extraction, 425-432

Stable isotopes, 441-451

Standard dilution method, 75-88

Suess effect, 57-65

Teeth, 235-242

Teotihuacán, 159-176

Transition dating, 39-56

Tree rings, 27-30, 257-263

Tube cracker, $177-178$
Uzbekistan, 235-242

Volcanic gas, 211-220

Waton, 181-192

Wiggle matching, 135-146

Xia dynasty, 21-25

Xinzhai site, 21-25

Yalâ (Yemen), 147-158

Zeolite molecular sieve, 441-451 